\title{
El desarrollo del castellano de los hablantes indígenas amazónicos en la escuela limeña*
}

\author{
Alicia Nidia Alonzo Sutta, Minie Lozada Trimbath, \\ Pedro M. Falcón Ccenta \\ Departamento Académico de Lingüística
}

\section{INTRODUCCIÓN}

IF 1 presente trabajo tiene por objeto describir la concordancia en la frase בn nominal (FN) y frase verbal (FV) en castellano por parte de los alumnos bilingües cuya lengua materna es una lengua indígena amazónica y el castellano, su segunda lengua. Estos escolares asisten a la escuelas de Lima. donde la enseñanza se realiza en castellano; por ello, hemos considerado pertinente abordar tal estudio, centrando nuestra atención en la producción escrita, la misma que está vinculada sustancialmente al desarrollo académico escolar.

\section{Biblioteca de Letras}

La inmigración deopoblacionésinildigenas/de 4 a amazonía a las grandes ciudades se viene incrementando en la últimos décadas. Lima y Huancayo son algunas de las ciudades que han recibido el influjo de estas poblaciones sea por la cercanía a las zonas geográficas de origen, así como por "mejorar sus niveles de vida”, o quizá también por razones de seguridad por la situación sociopolítica vivida en lás últimas décadas. Sea cual fuere la causa, muchas familias indígenas amazónicas se encuentran asentadas en los puntos marginales de la ciudad de Lima.

* Investigación que forma parte del proyecto "El desarrollo del castellano de los hablantes indígenas en la escuela limeña" ejecutado en 1999 por los profesores Meneses, Lozada, Alonzo, Pineda y Falcón, el cual comprende el estudio de hablantes indígenas amazónicos $y$ andinos. 
El traslado y retorno de los miembros de la familia del lugar de origen a Lima es frecuente y obedece al proceso mismo de la inmigración, en el cual se encuentran involucrados los niños en edad escolar, quienes junto a sus padres sufren muchos cambios, particularmente en su vida escolar.

El reciente proceso le da a las escuelas limeñas un nuevo carácter: diverso, multiétnico y multilingúístico; sin embargo, esta situación aún no es percibida por los directores de los centros educativos, reflejándose el desconocimiento de la presencia de alumnos bilingües amazónicos en el centro escolar que dirigen, y la idea errada de concebir una población escolar inmigrante andina o hijos de inmigrantes solamente andinos.

Cabe aclarar sin embargo que si bien se viene produciendo inmigración de familias indígenas amazónicas, existen aún pocos alumnos bilingües de lengua indígena-castellano en las escuelas de Lima, que creemos podría incrementarse en los próximos años.

La investigación contempla en primer término los datos sociolingüísticos de los Asentamientos Humanos donde se desarrollaron los estudios de casos, para lo cual consideramos datos relativos a la ubicación geográfica, composición poblacional, grupos indígenas, centros educativos, etc.; así mismo, detallamos la metodología utilizada, tanto en cuanto se refiere al estudio de casos como las de variables dependientes independientes, así como el proceso de recopilación "Jo información.

Por otro lado, en lo que respecta al análisis, damos cuenta de manera descriptiva parte de los resultados de la investigación; para ello, en base a un corpus seleccionado, se presentan las evaluaciones de los casos bajo el rubro concordancia en la FN y FV y Tiempos verbales.

\section{REAJUSTES}

Durante la ejecución del proyecto, tuvimos algunas dificultades que motivaron un reajuste de nuestro estudio desde el punto de vista metodológico. Es así que debido al número reducido de migrantes indígenas amazónicos en las escuelas visitadas y su relativa dispersión espacial en Lima, los casos estudiados fueron uno del nivel primario y cuatro del nivel secundario. 
De otra parte, no se realizó entrevistas formales a los profesores de aula donde estudiaban los niños seleccionados, pues asumían una actitud defensiva, tal vez, creemos, se sentían examinados o evaluados, razón por la cual la recolección de información fue mas bien indirecta, es decir, fruto de la conversación informal y observación.

Finalmente tuvimos la necesidad de la inclusión en el estudio de alumnos monolingües castellanohablantes de los mismos grados y de los mismos centros educativos de los casos ya mencionados como grupo referencial, hecho que además permitió no generar de ninguna manera la discriminación de los alumnos indígenas amazónicos.

\section{REGISTRO DE DATOS SOCIOLINGÜÍSTICOS}

Examinamos a continuación los datos sociolingüísticos referidos a las zonas donde se llevaron a cabo-los estudios.

\subsection{Asentamiento Humano "Horacio Zevallos Gamez"}

Está ubicado a la altura def kilómetro 16.5 de la carretera central, a la margen derecha, cerca al AA.HH. "Huaycán". Pertenece a la provincia de Lima, departamento de Lima. Fue creado el 27 de Sctubre de 1984.

\section{"Jorge Puccinelli Converso"}

La composición poblacional del Asentamiento concentra mayoritariamente pobladores andinos, provenientes de Ayacucho, Apurimac, Cuzco, Puno y Cajamarca; en menor proporción pobladores indígenas amazónicos y ribereños provenientes de Ucayali, Satipo y Chanchamayo del departamento de Junín, San Martín y Amazonas. La población indígena amazónica corresponde a los grupos Ashaninka, Shipibo, Yanesha, Machiguenga y Bora. El número de familias por grupo étnico se distribuyen como sigue:

$\begin{array}{lr}\text { Shipibo } & 11 \text { familias } \\ \text { Ashaninka } & 8 \text { familias } \\ \text { Yanesha } & 2 \text { familias } \\ \text { Machiguenga } & 1 \text { familia } \\ \text { Bora } & 1 \text { familia }\end{array}$


- Del total de familias indígenas sólo 2 estudian actualmente en el nivel primario y dos en el nivel secundario. Dos son shipibos y uno, hijo de padre andino y madre ashaninka, con manejo incipiente de la lengua Ashaninka.

Además, se observó la presencia de una familia constituida por padre mestizo y madre Ashaninka, cuyos hijos tienen como lengua materna el castellano. Sólo la madre habla Ashaninka.

En el Asentamiento existe un centro educativo inicial y otro de primaria y secundaria, denominado C.E $\mathrm{N}^{\circ} 1237$ Akira Kato, pertenece a la USE 06 Ate-Vitarte, creado el 26 de febrero de 1986. Existen además, tres centros educativos particulares, de inicial, primaria y secundaria, respectivamente.

El número de alumnos indígenas amazónicos alcanza a 6 , distribuidos 4 en primaria y 2 en secundaria, de los cuales sólo dos hablan su lengua materna. Ambos son del grupo etrolingüístico Shipibo.

En cuanto a los profesores debemos señalar que el $60 \%$ son contratados, muchos de ellos de origen andino, pero todos castellano hablantes.

Es importante destacar el desconocimiento absoluto de la presencia de alumnos de origėn indígena de la amazonía peruana por parte de los representantes de los centros edueativos xisitados, tan es asígue cuando establecimos las primeras coordinaciones con el Director del centro educativo Akira Kato se nos dijo que no había ahumnos indígenas de la amazonía y que la mayoría eran provenientes de poblaciones andinas, muchos de ellos de padres quechuahablantes.

\subsection{Casa indígena Tarata}

La Casa indígena Tarata se encuentra en Barrios Altos-cercado de Lima, a la altura de la cuadra 9 de la Avenida Grau, creada el 11 de junio de 1999. Está constituida por 18 familias, todas ellas corresponden al grupo etnolingüístico Shipibo. Si bien este número puede variar ascendentemente debido a visitas temporales de otras familias o personas que están de paso por Lima, en algunos casos puede ocurrir que una que otra familia se quede permanentemente en la Casa, así como algunas decidan regresar a sus lugares 
de origen (con menor frecuencia) o se trasladen a algún Asentamiento Humano en los alrededores de Lima.

La Casa Indígena además de servir de vivienda, sirve de hospedaje para indígenas, especialmente shipibos que llegan a Lima para realizar gestiones diversas. En ella funciona un aula de educación inicial sin valor oficial para niños de 2 a 5 años, los mismos que son hijos de los propios indígenas; la profesora no es indígena y desarrolla sus actividades en castellano.

El funcionamiento de la Casa Indígena está a cargo de organismos no gubernamentales.

Los centros educativos donde se forman los hijos de los indígenas shipibos son el colegio "República del Brasil" $\mathrm{N}^{\circ} 1032$, ubicado en Barrios Altos, cercado de Lima y el colegio "Villa los reyes" ubicado en el complejo comunal "Villa los Reyes" Ventanilla.

En el primer colegio mencionado sólo estudian dos niños, en segundo y tercer grado. Justamente el niño de tercer grado fue considerado en nuestro estudio. En el segundo colegio, consideramos un niño de primero de secundaria, el único estudiante indígena amazónico de este centro educativo. Respecto a este último caso, debemos manifestar que en el último trimestre del año, toda la familia se trasladó al Complejo Comunal "Villa los Reyes" según nos dijeron por la cercanía al colegio donde estudia el niño. Este hecho dificultó nuestro estudio, pưes pafadójicamente er '̂fiño fue irregular en su asistencia al colegio y por la distancia y desconocimiento de la casa donde vivía la familia nos fue difícil establecer coordinaciones con los padres.

Todos los integrantes de las 18 familias y todas aquéllas que llegan temporalmente a la Casa Indígena se comunican entre ellos en su lengua materna, es decir, en Shipibo.

\section{METODOLOGÍA}

El estudio del castellano de alumnos indígenas amazónicos realizado comprende las siguientes etapas y acciones. 


\subsection{Determinación de los casos}

\subsubsection{Ubicación de alumnos}

La información sobre alumnos de selva que estudian en Lima se obtuvo de diversas maneras.

Contacto con nativos de la Amazonía y con especialistas en el estudio de dicha área

De un lado, personas nativas de la selva que radican en el asentamiento Humano Horacio Zevallos nos indicaron que en dicho lugar viven varias familias de la selva que tienen niños en edad escolar. De otro lado, y gracias a un especialista, se tuvo conocimiento de la Casa Indígena, lugar donde se alojan familias que llegan a Lima de la selva y que se encuentra situada en el jirón Tarata, en los Barrios Altos.

Contacto con directores y subdirectores de centros educativos nacionales

Se visito colegios en donde se hos había indicado que estudiaban niños indígenas amazónicos. En ellos, se entrevistó a algunas autoridades educativas para que nos ayudaran a ubicar a estos alumnos. A través de las entrevistas, constatamos que de los tres colegios, dos no tienen datos estadísticos sobre la procedencia de sus alumnos. Las autoridades con quienes conversamos son:

- $\quad$ El Director del "C.Ë "Akira Kato", Sección secundaria, ubicado en el asentamiento humano Horacio Zevallos.

- El Director y la Subdirectora del C. E. República del Brasil, sección primaria, ubicado en Barrios Altos.

- El Subdirector del C.E. Villa Los Reyes Areas Técnicas, Ventanilla.

Conversación con profesores de escuelas nacionales

En los colegios, profesores de algunas de las áreas nos dieron facilidades para entrar en contacto con sus alumnos (profesores de ciencias naturales, inglés, orientación y bienestar del educando). A pesar de nuestros esfuerzos, no logramos el apoyo de los profesores de lenguaje como hubiera sido deseable. 


\subsubsection{Selección de casos}

Tuvimos dificultad para encontrar alumnos bilingües que cursaran el sexto de primaria y el quinto de secundaria tal como había sido inicialmente planificado. Sin embargo, como sí encontramos algunos alumnos procedentes de selva, de ellos, seleccionamos aquellos casos que se acercaban más a nuestra propuesta original, decidiéndonos por los que cursaban el año inicial y el año final de la secundaria. Aunque no estaba planificado, un alumno bilingüe de tercero de primaria también fue incluido debido a que reunía características lingüísticas y educativas de particular interés para nuestra investigación.

Pero no sólo nos hemos limitado al estudio de los casos de niños de la selva, sino que en el primer año de secundaria hemos tenido la facilidad de contar con un grupo de alumnos que no proviene de la Amazonía. La finalidad de trabajar con este segundo grupo ha sido tenerlo como referencia para conocer si el dominio de lengua que se tiene en el grado es o no similar en ambos grupos, el que es de selva y el que no lo es.

\subsubsection{Alumnos de la Amazonía seleccionados}

\section{Primer Año de Secundaria}

\section{(a) Alumno J.D.U. Biblioteca de Letras "Jorge Puccinelli Converso"}

Edad y lengua: Tiene 13 años de edad, es natural de Yarinacocha, Pucallpa, y es bilingüe shipibo-castellano.

Familia: Los padres son de Pucallpa y pertenecen a la etnia shipibo. El alumno ha vivido en Yarinacocha al igual que su hermano que estudia actualmente en el mismo colegio.

Estudios del alumno: Ha realizado casi todos sus estudios en el C. E. Akira Kato, en el asentamiento humano Horacio Zevallos. El año 1998 estuvo estudiando en la selva, en Pucallpa, y luego en el 99 regresó a Lima.

En el colegio tiene problemas con el curso de Lenguaje y con el de Religión (la familia del niño es de religión evangélica. 
(b) Alumno P.R.N.

Edad y lengua: Tiene 12 años. El niño es bilingüe shipibo-castellano.

Padres: Los padres son procedentes de la selva y son bilingües. En casa emplean predominantemente el shipibo.

Estudios del alumno: C.E. Villa Los Reyes Áreas Técnicas, Ventanilla. Los padres consideran que necesita ayuda en matemática.

\section{Cuarto Año de Secundaria}

(c) Alumno W.D.U.

Edad y lengua: De 18 años de edad, y natural de Pucallpa, es bilingüe shipibo-castellano. Es hermano del alumno de $1^{\circ} \mathrm{Sec}$. J.D.U. antes mencionado. Como él, ha vivido también en Yarinacocha antes de venir a Lima.

Padres: Son de Pucallpa y pertenecen a la etnia shipibo.

Estudios del alumno: Los dos primeros grados de primaria los estudió en Pucallpa pero a partir de tercer grado lo hizo en Lima. Asiste al C.E. Akira Kato desde sexto de primaria hasta la actualidad en que cursa el cuarto de secundaria.

\section{Quinto Año de Secundaria}

\section{"Jorge Puccinelli Converso»}

(d) Alumno R.M.S.

Edad y lengua: Nació en la comunidad nativa de Marankiari. El alumno es hablante de castellano pero dice conocer algunas palabras sueltas en

Padres: El padre es arequipeño y vivió en la selva desde su adolescencia. La madre es de Chanchamayo pero sabe poco ashaninka. La abuelita quien falleció hace dos años sí hablaba dicha lengua. El alumno manifiesta su interés por esta lengua y con ayuda de sus tíos está estudiándola aunque por ahora conoce solamente algunas palabras. 
Estudios: Estudió hasta cuarto de primaria en la selva en un colegio adventista de la $\mathrm{C} \mathrm{N}$ de Marankiari en donde todas las clases se desarrollaban en castellano. Luego vino a Lima.

Actualmente manifiesta estar regular en sus estudios siendo Literatura el curso que prefiere.

\section{Tercer Grado de Primaria}

(c) Alumno H.R.V.

Edad y lengua: Tiene 11 años y es bilingüe shipibo-castellano

Padres: Son de la etnia shipibo y son bilingües.

Estudios: Estudia en el C.E. República del Brasil. Es un niño muy vivaz e inclinado al dibujo.

\subsubsection{Alumnos seleccionados con fines de referencia}

Como ya se ha mencionado, tanto en el C.E. Akira Kato como en el C.E. Villa Los Reyes se consideró un grupo de siete alumnos en total, del primer año de secundaria, que no provienen de la selva, con el fin de tener una referencia respecto deb manejo del castellano que en ese grado tienen los alumnos.

\section{"Jorge Puccinelli Converso"}

En el Akira Kato, las edades de tres niños oscilan entre 12 y 13 años. Dos han nacido en Lima y uno en la sierra. En un caso los dos padres son de la sierra, en el caso de los otros dos niños, uno de los padres es de la costa (Lima e İca). En cuanto a dominio de lenguas, un alumno habla sólo castellano, otro entiende quechua pero no lo habla, mientras que un tercero sólo entiende algunas palabras en quechua.

En el Colegio Villa Los Reyes, las edades son semejantes a las anteriormente mencionadas. En cuanto a la procedencia, tenemos niños que han nacido en la sierra, por ejemplo, en Cajamarca, y otros, que son de Lima. La lengua que dominan es el castellano. 


\section{ELABORACIÓN DEL INSTRUMENTO}

Antes de elaborar el instrumento para estudiar el castellano indígena amazónico, se recolectó información sobre el uso de lengua castellana que los profesores esperan del nin̄o en la escuela. La recolección de esta información se realizó por medio de conversación con algunos profesores, con los padres, y con los mismos niños. Además se hizo revisión de cuadernos, y se pidió a los niños, en algunos casos que leyeran, y, en todos los casos, que escribieran un texto breve descriptivo o narrativo. Es en este aspecto en donde podría habernos sido de utilidad que los profesores de lengua nos ayudaran, pero como ya se indicó esto no fue posible. En uno de los casos, también nos fue posible realizar observación de clase.

Algo que facilitó en parte el análisis de cuadernos y libros, y la conversación con los alumnos, en dos oportunidades, fue la ausencia del profesor en la clase que le correspondía conducir.

Observamos que los cuadernos de secundaria de lenguaje, matemática y ciencias naturales presentan cuestionarios y ejercicios controlados por el profesor. En ellos se observa que el alumno copia o reproduce el lenguaje de ciertos párrafos de los libros o de la pizarra. Probablemente en algunos casos toma dictado. En el primer año de secundaria, no se pudo observar escritos en donde se manifiesta la lengua en sü uso ereativo. Sabemos que sí se les pedía que escribieran composjciones y poemas, pero los papeles donde están estos escritos no los tienen los alumnos sino el profesor.

En el caso del tercer grado de primaria, el libro del Ministerio de Educación que se usa, propone al alumno alternativas para crear sus textos. A pesar de ello, y probablemente porque se manda hacer este tipo de tarea en la casa, el alumno sin apoyo se siente desorientado y tiende, no a crear sino a reproducir algo leído antes.

\section{CARACTERÍSTICAS DEL INSTRUMENTO}

La prueba elaborada para examinar la sintaxis del castellano de los alumnos amazónicos es la misma que se ha empleado para los alumnos de procedencia andina. Dicho instrumento consta de las siguientes partes: comprensión de texto, análisis de estructuras sintácticas y redacción. 
El pasaje de lectura (de carácter narrativo) trata un tema relacionado con la Amazonía, el mismo que está constituido por cuatro párrafos breves, cuyo grado de complejidad es mínimo; a continuación se consignan preguntas sobre el contenido que piden información sobre el título, los personajes y las ideas principales del texto. Se busca conocer si el alumno maneja suficiente castellano como para entender lo que lee en u $\mathrm{n}$ texto.

La parte de análisis de estructuras comprende tres secciones, una de ordenamiento de palabras para construir oraciones, otra de opción múltiple sobre preposiciones, y la tercera, oraciones para completar con la preposición adecuada. Estas preguntas miden el dominio que tiene el niño de aspectos de sintaxis.

Para la parte de producción escrita, se emplea un estímulo visual que presenta una situación a partir de la cual, el alumno debe construir un cuento. Ésta será analizada en algunos aspectos, principalmente concordancia .

Tanto el pasaje de lectura como la lámina empleada como estímulo para la redacción, pertenecen a libros de cuentos publicados.

\section{APLICACIÓN DEL INSTRUMENTO.}

La aplicación de Ra prueba se realizó- en los colegios Akira Kato y Villa Los Reyes. Por razones terinasistenciaed lia escuela, Sen dos casos, se tuvo que hacer en el domicilio de los alumnos.

El tiempo para desarrollar la prueba tomó desde veinte minutos en el caso de los alumnos que no proceden de selva hasta cuarenticinco minutos en el caso de aquéllos que sí proceden de ella. El niño de tercer grado de primaria tomó aproximadamente una hora.

\section{CONCORDANCLA}

\subsection{En la Frase Nominal}

En la estructura morfosintáctica y sintáctica del castellano, la frase nominal está constituida de un elemento nuclear, es decir, el nombre o el 
pronombre y como elementos complementarios las distintas clases de determinantes que posee la lengua castellana: mi, la. estos, alguna, pocos,... etc.

En relinción al núcleo de la FN, sabemos que éste es quien determina el género y el nímero de los elementos que actúan como sus modificadores directos, llámese determinante o adjetivo.

En la siguiente descripción daremos cuenta de las dificultades que tienen los bilingües para estructurar la frase nominal en castellano. Sean éstos relacionados con los determinantes así como también con los nombres

- El género

El castellano posee un morfema que expresa género; si éste es masculino entonces el morfema pertinente sería -o. Cuando el nombre es de género femenino le corresponde el morfema flexivo -a.

Cuando la frase nominal presenta determinantes, éstos concuerdan en género y número con los nombres; sin embargo en los siguientes enunciados, referidos por los hablantes bilingües, se puede observar una inseguridad en el manejo de la concordancia entre determinantes y nombres. Ejemplos:

alumno

...una puma macholioteca de IPRtras

...por el selva adentro. Puccinelli CoPR ${ }^{\text {erso" }}$

...y los persona que grita

...todos las persona...

$P R$

...qué hacer con este estrella...

$H R$

...no a otro persona...

...todos los cosas malas

$H R$

$J D$

$J D$

- $\quad$ El número

En el primer enunciado se observa la propuesta del nombre pluralizado con el morfema -s correspondiente al número en castellano, lo que nos indica que nuestro entrevistado reconoce el morfema de número, más aun se confirma con la presencia de un adjetivo en completa concordancia con el número y género del nombre ojos tapados; sin embargo, cuando éste emplea un determinante posesivo se produce un error de concordancia en el número
plural Ejemplo: 
El precario uso de concordancia entre el núcleo y sus determinantes se puede apreciar en los ejemplos planteados por nuestro entrevistado PR.

La descripción de la frase nominal, de las primeras cuatro frases nominales, el alumno bilingüe emplea el nombre en singular por lo que se esperaría que los determinantes también presenten la misma característica morfosintáctica; sin embargo se presenta de la siguiente manera:

...pero las persona morian... $\quad P R$

...pero tres persona quedaron... $\quad P R$

...los que ...fueron las puma... $\quad P R$

\section{- Otros casos en la FN}

Presentamos otros casos como la omisión del determinante en la siguiente frase nominal compleja: la mamá de Pepe. Esta pequeña dificultad podría estar vinculada a factores de tipo léxico semántico. Sabemos que el alumno conoce y emplea los artículos definidos puesto que en ese mismo enunciado se observa la FN : los vecinos; sin embargo el término de parentesco N: mamá, no presenta deferminante. ¿ A qué se debe esta mínima omisión? Nuestro próxima inyestigación quizá dé cuenta de esta interrogante

...y Ø mamá de Pepeglıamócaitoslliecinos...rso HR

De otro lado el mismo entrevistado demuestra el empleo del artículo definido al proponer la siguiente $\mathrm{FN}$ :

...el pepe...

$H R$

A pesar que en dicho contexto, cuando se encuentran nombres propios no se requiere la presencia de los determinantes.

Los siguientes enunciados presentan por un lado la doble presencia de determinantes, así como del nombre en forma inconclusa, característica del entrevistado JD. ¿El alumno no conoce los morfemas flexivos de género y número? 


\subsection{En la Frase Verbal}

Esta sección dará cuenta no sólo de la falta de concordancia entre sujeto y predicado, unidades básicas de una oración, sino además de los mecanismos que los alumnos bilingües utilizan con la finalidad de comunicarse a nivel escrito.

A) Las características lingüísticas que a nivel escrito presenta nuestro entrevistado JD se manifiesta, como bien dijéramos antes, en la escritura de palabras verbales y nombres fundamentalmente. Probablemente el poco dominio de las flexiones que requieren estas unidades, los lleva a expresar verbos y sustantivos incompletos provocando las discordancias con los elementos de su entorno, sean determinantes en la frase nominal; sean complementos en la frase verbal. cultades:

A continuación, presentamos los enunciados que ofrecen mayores difi-

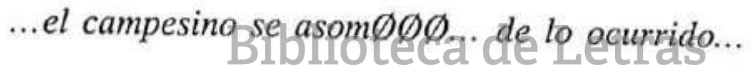

Debemos suponef que el escudiante bilingure, según el tiempo verbal del relato narrado, debió colocar bró constituyéndose el verbo asombró; sin embargo, no podemos suponer qué tiempo verbal y qué persona gramatical habría expresado el estudiante. Posiblemente la duda acerca de estas dos características, además de otras que se expresan en el verbo, lo llevan a plantear el verbo asom que a continuación prresentamos ejemplo:

En el enunciado se puede confirmar lo señalado, a través del siguiente ...en un pueblo ... avitava $\emptyset$ unas person ...

Además de la dificultad señalada anteriormente, en relación a la escritura incompleta de las palabras, JD transfiere el orden sintáctico de los cons- 
1 En un pueblo $\mathrm{CC}$

2 Avitava Verbo

3 Unas person Sujeto

...unas person avitava en un pueblo

$\mathrm{S} \quad \mathrm{C}$

Otro elemento digno de observar es el verbo, el mismo que al presentarse en tal forma avitava está expresando la acción ejecutada por un sujeto singular, mas el sujeto expresado es plenamente en plural.

La dificultad de JD al expresar los verbos incompletos, en castellano, le provoca dificultades a nivel de producción escrita, esto mismo también puede generarle dificultades de comunicación a nivel de comprensión escrita, mucho más aun si consideramos que este alumno se encuentra cursando el primer año de secundaria.

B) El siguiente caso corresponde al estudiante bilingüe PR, quien actualmente cursa el primer ano de secundaria Nuestro entrevistado, en forma semejante al anterior, muestra la omisión de un segmento en los verbos; no obstante la ausencia de dicho segmento, el enunciado logra la significación semántica pretendida.

... los que escucharoø... "Jüeca de Letras

...fueron los pumas que comía $\emptyset \ldots$

El segmento nasal /n/ es omitido en ambos verbos, sin embargo el tiempo y la persona gramatical han sido considerados por el alumno y se percibe a través del morfema flexivo que en forma parcial está expresado en cada verbo.

Aparentemente el caso de PR no muestra dificultades a nivel de la oración, sin embargo debo indicar que entre los relatos de los estudiantes bilingües que presenta mayor dificultad para expresar ideas, el de PR es el que destaca.

C) Nuestro entrevistado es el alumno de primaria HR, bilingüe shipibo/ castellano, quien presenta algunas discordancias entre el sujeto ejecutor de la acción y el verbo. Ejm. 
...Pepe y su mamá lo vio.... (a la estrella)

...unos amigos le dijo no bañas... (al niño)

Se espera que ante un sujeto plural, ejecutor de la acción, se complemente con un verbo que concuerde con aquél en numero y no sólo en persona; sin embargo en ambas oraciones se observa la discordancia referida a número.

De otro lado, también podemos suponer que la llamada discordancia de número esté expresando, para el niño, una total concordancia del verbo con el objeto directo

vio (la estrella)Objeto directo $=$ lo vio

dijo (al niño)objeto indirecto $=$ le dijo

Pero, debemos señalar que un corpus más amplio permitiría arribar a conclusiones más reales de este aspecto sintáctico.

\section{TIEMPOS VERBALES}

Los bilingües al dominar en grado diferente las lenguas que conocen, y al presentar diferencias, a veces marcadas, entre el uso de la lengua oral y la escrita, presentan, entre-otros aspectos, usos más o menos adecuados de los tiempos verbales. Una primera aproximación al dominio de estos tiempos que tienen los alumnos es el objetivo de esta sección.

Para realizar el estudio de los tiempos verbales el material que se analizó es el cuento o leyenda que los niños escribieron como parte de la prueba. Nos vamos a centrar en los dos casos de primero de secundaria ya que en dicho año de estudios contamos, no sólo con alumnos de procedencia amazónica, sino también con un grupo procedente de otras zonas que nos sirve de referencia.

El análisis va a considerar el empleo del tiempo del verbo dentro de la totalidad del texto escrito por el alumno. Se busca conocer si los niños establecen la correspondencia o relación de los tiempos de manera adecuada. Debemos precisar que en esta sección no estamos analizando concordancia de número y persona, ni ortografía. 
El procedimiento empleado consiste en ubicar, primero, los verbos usados en el texto (están excluidas las formas no personales), luego se revisa si el tiempo de cada uno de ellos es el adecuado, a continuación se determina el número de verbos que aparecen señalando el tiempo al que pertenecen, y finalmente, se presenta la frecuencia de los verbos usados de manera adecuada y los de manera inadecuada.

A continuación presentamos los resultados de los dos alumnos amazónicos de primero de secundaria seguido de los resultados de tres de los niños del grupo de referencia.

\section{CASOS}

(a) Alumno P.R.N.

Téngase en cuenta que este alumno es bilingüe shipibo-castellano y aunque vive en Lima en su casa la familia habla predominantemente shipibo.

El escrito de este alumnop presenta 26 verbos. Recordemos que no se está considerando errores de concordancia y de ortografía como sería el caso en los siguientes ejemplos: "....cada uno agaron una flecha..., ...los persona se dedicaron..., etc. Lo que interesa en este caso es si la idea del tiempo del verbo que se escribe es la adecuada para esa parte del texto. «Jorge Puccinelli Converso»

De los 26 casos, en 25 el tiempo es usado de manera adecuada:

Verbo

Frecuencia

Modo indicativo:

Presente

Pretérito indefinido

Imperfecto

Total de ocurrencias:

25

En cuanto al uso inadecuado, de 26 casos encontramos 1 , como se observa a continuación: 
“...cada uno agaron una flecha que era venenosa y cada uno se les tiro y no les dava a la puma macho por que saltava de arbol para que comere (?) gentes que teraron flechas..." (¿cada uno agarró?, ¿cada uno ...tiró?, igentes que tiraron?)

Verbo

\section{Frecuencia}

Forma comere

no adecuada.

El alumno presenta un $96 \%$ de uso correcto de los tiempos verbales.

(b) Alumno J.D.U.

El alumno es también bilingüe shipibo-castellano y ha hecho todos sus estudios, excepto el año 1998, en Lima.

El texto escrito por este alumno presenta 33 verbos y una omisión. te.

A continuación vemos que de los 33 verbos 30 son usados adecuadamen-

$\begin{array}{lc}\text { Modo IndicativBiblioteca de Letras } \\ \text { Presente «Jorge Puccinellizanverso» } \\ \text { Pluscuamperfecto } & 1 \\ \text { Futuro } & 3 \\ \text { Pretérito perfecto } & 2 \\ \text { Imperfecto } & 11 \\ \text { Pretérito indefinido } & 10\end{array}$

Total: 30 ocurrencias

En cuanto al uso inadecuado observamos:

Imperfecto 1 (omisión)

“... Por la comunidad que se convertio en una laguna era pegado ( $i$ pecadora?) havio (¿había?) violencia todos las cosas malas (¿eran malas?)." 
Pretérito indefinido 3 (un tiempo en vez de otro, y problema que parece de ortografía)

"El campesino se asom de lo que avia sucedido fue contento a su casa a traer erramientas ..." ( ¿se asombró?)

"Llegando al pueblo se dia la sorpresa que su pueblo se transfordo en forma de estrella." (¿se dio la sorpresa?, ¿se había transformado?)

El alumno presenta un $91 \%$ de uso adecuado de tiempos verbales.

\section{GRUPO DE REFERENCIA}

En el C.E. Akira Kato, al analizar el uso de tiempos verbales de los compañeros de J.D.U., los mismos que no son indígenas amazónicos, observamos lo siguiente:

El alumno A. P., cuya madre es de Lima y cuyo padre, de la sierra, emplea 19 verbos en su cuento.

Él emplea de manera adecuada 17 verbos ( $89.47 \%$ ) los cuales se distribuyen como sigue:Biblioteca de Letras

\section{"Jorge Puccinelli Converso»}

1 uso impersonal, presente de indicativo

10 pretérito indefinido, indicativo

5 presente, indicativo

1 presente de subjuntivo

En cuanto a uso inadecuado observamos:

2 imperfecto, en vez de pretérito

Un caso que destaca es el de la alumna E.E. cuyos padres son bilingues quechua-castellano. La niña no tiene errores en su uso de los tiempos verbales. Según la alumna ella no sabe sino unas cuantas palabras sueltas en quechua. De las 22 ocurrencias tenemos 22 usadas adecuadamente, esto es el $100 \%$. 
18 imperfecto, indicativo

2 presente de indicativo

2 presente de subjuntivo

En el caso del alumno E. M., hijo de una persona de la costa (Ica) y una de la sierra, sí se observa poco control de los tiempos verbales. Al narrar, hace uso del presente lo cual podría estar bien si no fuera porque de pronto se encuentran cambios a otros tiempos verbales que no corresponden. Como hay mucho uso inadecuado es difícil calcular el porcentaje.

Comparando ambos grupos observamos lo siguiente:

Uso adecuado de los alumnos amazónicos

P.R.N.

$96 \%$

J.D.U

$90 \%$

Uso adecuado de los alumnos del grupo de referencia
A:P:
$89.47 \%$
E:E.
$100 \%$
E.M.
no ha sido posible el cálculo

En cuanto al control de los tiempos verbales vemos que la situación entre los dos grupos no presenta diferencias muy grandes. En otras palabras, los alumnos amazónicos no tienen difíeultades mayores que los demás aunque no tenemos a nadie conpr100\%udeiusolidecuados. Donde sí parece haber grandes dificultades es en la concordancia, pero para informarse de esto hay que remitirse a la sección correspondiente.

\section{CONCLUSIONES}

1. Los alumnos indígenas amazónicos reproducen en la lengua escrita errores producidos en lengua oral. En términos sintácticos y morfosintácticos se cometen errores de concordancia en la $\mathrm{FN}$, entre DET. y N; N-ADJ.; FN _ FV; y dependiendo de las características del verbo, esto es, si requiere de atributo o complemento pronominal, también ocurre en la FV. 
2. Se observa uso del sufijo -mente para adverbializar cualquier adjetivo. Ejemplos: arrodillado + -mente $\rightarrow$ arrodilladamente asustado + -mente $\rightarrow$ asustadamente.

Quiere decir que el hablante aplica la regla que opera en el castellano, pero de manera generalizada, sin tomar en cuenta las restricciones que éstas tienen por lo menos en la gramática normativa.

3. Aunque no forma parte del análisis del tema central del proyecto, entre otras observaciones, debemos mencionar el uso de los tiempos verbales. Los hablantes indígenas amazónicos en términos generales no tienen mayor deficiencias para el uso escrito de los tiempos verbales, diríamos que no se establece diferencias mayores en relación al grupo de control o referencia; sin embargo se observa el uso de un número reducido de verbos en tanto variación léxica y conjugaciones complejas.

Asi mismo cabe indicar la presencia de dificultades significativas de carácter fonético-fonológico, léxico y textual.

4. E! uso de las conjunciones en lengua escrita por parte de los alumnos indígenas amazónicos se enmarcan dentro del concepto de unidades de relación que reúnen elementos lingüísticos dentro de una misma categoría. Sin embargo se obseryan algunas dificultades que parece tienen origen en los enunciados inadecuadamente construidos. Ejemplo:

... desesperado corio así su chacra si era cierto lo de su sueño. JD

Por ello, creemos que es necesario recopilar más textos que nos permitan incursionar en un análisis más exhaustivo. 


\section{BIBLIOGRAFÍA}

BOUQUIAUX, Luc y THOMAS, J.

1992

Studying and Describing Written Languages. A publication of the Summer Institute of Linguistics.

ESCOBAR, Anna María

1990

Los bilingües y el castellano en el Perú. IEP. Lima.

LANDA, Cinthia y URALDE, Isabel

1996

"La estrella que cayó del cielo", del libro Déjame que te cuente. Santafé de Bogotá D.C.

OSTROSKY-SOLIS, Feggy y ARDILA, Alfredo

1991

Lenguaje oral y escrito. D. Trillas. México.

TIPACOM

1990

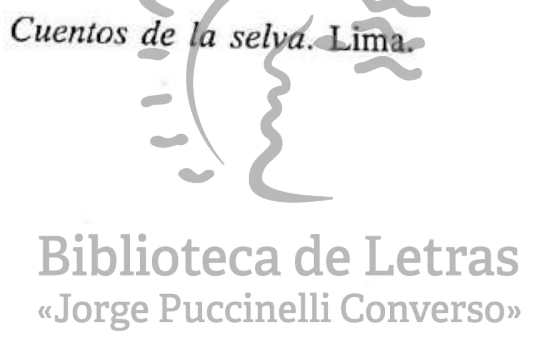

